Check for updates

Cite this: RSC Adv., 2019, 9, 36287

\title{
Room-temperature phosphorescent polymers with excitation-wavelength and delay-time emission dependencies $\uparrow$
}

\author{
Linmin Zou, ${ }^{a}$ Xiaojin Qin, ${ }^{a}$ Huazhen Sun, ${ }^{a}$ Shiqi Wang, ${ }^{a}$ Wenwen Ding, ${ }^{a}$ Yuanli Liu, ${ }^{a}$ \\ Chun Wei, ${ }^{* a}$ Bingli Jiang ${ }^{\star b}$ and Yongyang Gong (DD *a
}

Received 29th August 2019

Accepted 2nd November 2019

Novel room-temperature phosphorescent materials based on commercialized poly(4-styrenesulfonic acid-co-maleic acid) salt have been identified with aggregation-induced emission and room temperature phosphorescence emission characteristics. We systematically investigated their excitationwavelength and delay-time dependencies to provide new insight into the potentiality of these materials for multiple industrial applications, such as optical storage and anti-counterfeit labelling.

rsc.li/rsc-advances

\section{Introduction}

Room-temperature phosphorescent (RTP) materials have received increasing attention due to their unique photophysical processes and promising applications in photonics, organic electronics, bio-electronics, and anti-counterfeit labelling. ${ }^{1-6}$ However, the RTP materials are mainly limited to transition-metal- and rare-earth-ion-based inorganic materials or organic precious metal complexes because the presence of metals can promote the spin-orbit coupling. Normally, these RTP materials require rare and expensive elements such as rare earth metals, platinum, and iridium. Therefore, given the tedious preparation conditions and the cost of noble metals, the development of low-cost and processability rare-metal free organic RTP materials has been drawn extensive attention over the past years. Furthermore, it is well known that the triplet excitons are prone to nonradiative relaxations through vibrational stretching and external quenching (i.e., $\mathrm{O}_{2}$ ), making it challenging to achieve efficient RTP. Intensive effort has been made to reduce or eliminate vibrational stretching and external quenching of triplet excitons via chemical or physical methods. Physical methods can stabilize the triplet excitons in a rigid environment by suppressing nonradiative decay pathways to activate the RTP emission; typical physical methods include crystal formation, ${ }^{2,7-13}$ embedding into

${ }^{a}$ Guangxi Key Laboratory of Optical and Electronic Materials and Devices, College of Materials Science and Engineering, Guilin University of Technology, Guilin 541004, China.E-mail: 1986024@glut.edu.cn; yygong@glut.edud.cn

${ }^{b}$ College of Pharmacy, Guilin Medical University, Guilin 541004, China. E-mail: jiangbingli@foxmail.com

$\dagger$ Electronic supplementary information (ESI) available: EDX, LDS, CIE chromatic coordinates, phosphorescence emission with different delay time of PSSMA solid powders under different excitation wavelengths. See DOI: 10.1039/c9ra06863e rigid hosts, ${ }^{14-16}$ polymer assistance, ${ }^{17}$ metal-organic framework (MOF) coordination, ${ }^{18-20} \mathrm{H}$-aggregation, ${ }^{21-23}$ and many others. ${ }^{24-28}$ Chemical methods can promote spin-orbital coupling by incorporating functional groups such as heavy atoms, aromatic carbonyls. ${ }^{29-31}$

Currently, most studies focus on small organic compounds in the field of RTP materials. However, it is impossible for small RTP organic molecules to be used in ink-jet or roll-toroll printing for large-scale commercial production and applications. Thus, it is essential to develop novel polymeric RTP materials. There are two methods to obtain RTP polymeric materials. ${ }^{5}$ One approach is to incorporate organic phosphors into rigid polymer matrixes; the second method is to introduce a noble metal or a heavy atom in the main chain or side chain of the polymer to promote spin-orbit coupling. Although great effort has been devoted in the past few years, the development of noble-metal and halogen-atom free RTP polymer materials remains a formidable challenge. ${ }^{32-34}$ In our previous work, we demonstrated that ionic bonds restricted the molecular motions (rotations and vibrations), thus fabricating polymer materials that contained unconventional chromophores displaying persistent phosphorescent emission behavior at room temperature. ${ }^{35,36}$ The presence of ionic bonds, which have shorter bond length and higher bond energy than hydrogen bonds, halogen bonds, and other intermolecular forces, renders them more effective in reducing the molecular motions that, thus, negatively impact the non-irradiation and improve emission efficient. Indeed, the polydispersity of the molecular weight is one of the remarkable characteristics of polymers, which may form multiple emitting centres ${ }^{37-39}$ and emit multicolor RTP under different excitation wavelengths.

RTP polymer materials with excitation-dependent phosphorescence emission properties provide a promising alternative for anti-counterfeit security material. However, the so 
far reported RTP materials show two different emission colors under both UV on and UV off states and could not exhibit three or more colors; thus, they do not fulfill the requirements of modern anti-counterfeit applications. Recently, we observed that phosphorescence emission from cellulose depends on excitation wavelength at $77 \mathrm{~K}{ }^{40}$ Huang group reported that small organic compounds exhibit different RTP emission at room temperature. ${ }^{41}$ Nevertheless, few studies investigated the RTP materials with excitation wavelength dependence.

Herein, we report our recent progress in the fabrication of polymeric RTP materials from commercialized poly(4styrenesulfonic acid-co-maleic acid) (PSSMA) salt ionomer (Chart 1). These materials not only exhibit aggregation-induced emission activity and excitation wavelength-dependent emission color but also their phosphorescence is dependent on the delay time; in other words, the color of their phosphorescence activity is related to the length of time after the excitation light stops. To the best of our knowledge, this is the first investigation of the relationship between phosphorescent emission color and delay time at room temperature.

\section{Experimental}

\subsection{Materials}

PSSMA was obtained from J\&K Scientific., Ltd. Metal salts of zinc acetate dihydrate and calcium chloride hydrate were obtained from Adamas Reagent Co., Ltd. Distilled water was prepared by the Milli-Q Pure Water instrument. The commercially available reagents were used without further purification unless otherwise stated.

\subsection{Characterization}

UV-vis absorption spectra were obtained using a UV3600 UV-vis spectrophotometer at ambient conditions. The mean diameter $\left(D_{\mathrm{m}}\right)$ and polydispersity (PD) of PSSMA solution were determined on a Malvern Zetasizer nano ZS90 instrument equipped with a He-Ne laser $(\lambda=633 \mathrm{~nm})$ at a scattering angle of $90^{\circ}$ at $25^{\circ} \mathrm{C}$. X-ray diffraction (XRD) measurements were performed on an $\mathrm{X}^{\prime}$ Pert PRO X-ray diffractometer. The emission spectra, solids quantum yields, and lifetime were recorded on a FluoroMax-4 fluorescence spectrophotometer. Quantum yields $(\Phi)$ of solutions were estimated using 2-aminopyridine $(\Phi=60 \%$ in $0.1 \mathrm{~N} \mathrm{H}_{2} \mathrm{SO}_{4}$ ) as standard. ${ }^{42}$

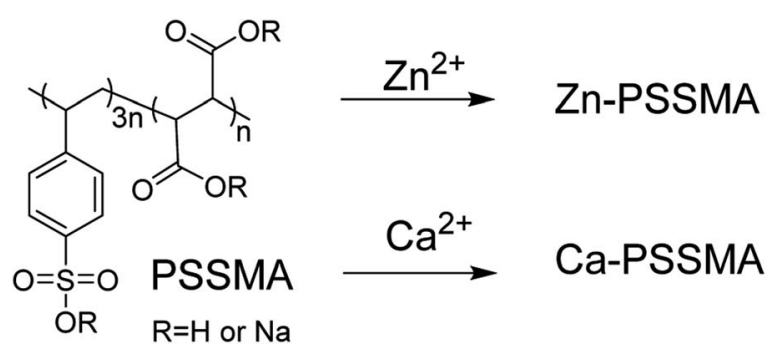

Chart 1 Chemical structure of PSSMA and synthetic routes to generate Zn-PSSMA and Ca-PSSMA.
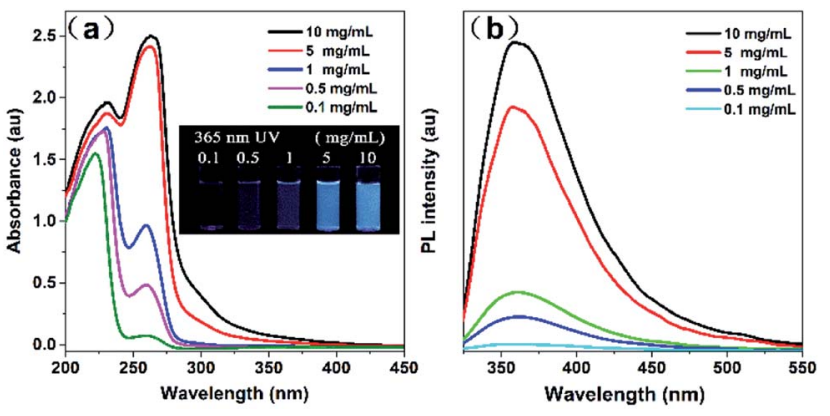

Fig. 1 The absorption (a) and fluorescence (b) spectra of the different concentrations PSSMA aqueous solutions at room temperature. Photographs in (a) are PSSMA water solutions with different concentrations taken under $365 \mathrm{~nm}$ UV light illumination. The excitation wavelength of the fluorescence spectrum is $300 \mathrm{~nm}$.

\section{Results and discussion}

PSSMA was purified by the solution-precipitation method. Firstly, a small amount of PSSMA was dissolved in water and then added dropwise into methanol via a cotton filter to precipitate this polymer. Both Ca-PSSMA and Zn-PSSMA were prepared by ion-change methods via $100 \mathrm{mg}$ PSSMA with $10 \mathrm{mg} \mathrm{Zn}^{2+}$ and $\mathrm{Ca}^{2+}$, respectively; then, they were purified by dialysis to remove excess zinc or calcium salt and then precipitated in methanol. Final products were characterized (Fig. S1, ESI†).

\subsection{Aggregation-induced emission (AIE)}

Fluorescence spectra and UV absorption spectra of PSSMA aqueous solutions with different concentrations were measured under room temperature. As shown in Fig. 1, the absorption intensity gradually increases, while the absorption wavelength gradually red-shifts as the concentration gradually increases due to the scattering effect caused by aggregated nanoparticles $\left(D_{\mathrm{m}}=5.75, \mathrm{PD}=0.29\right.$, Fig. S2, ESI $\left.\dagger\right)$. As shown in Fig. 2a, under the irradiation of a $365 \mathrm{~nm}$ UV lamp, the lowconcentration solution does not emit light, whereas the highconcentration solution emits significant light. Besides, the $\Phi$
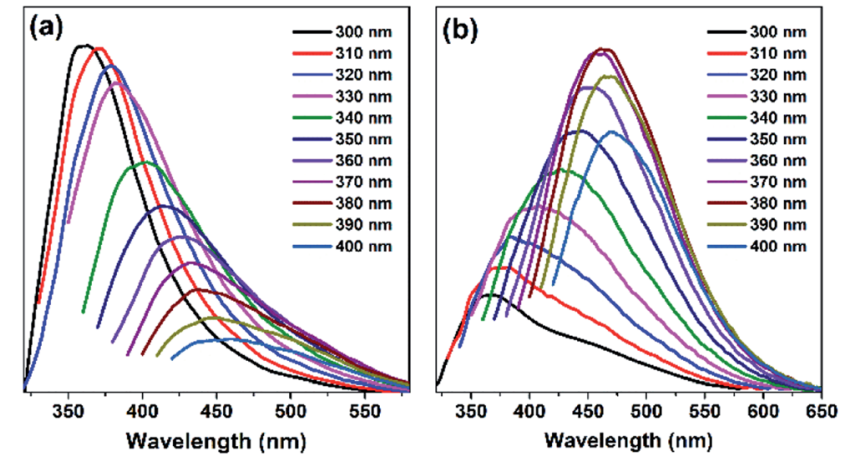

Fig. 2 The fluorescence spectra of PSSMA water solutions (a) and solids samples (b) with different excitation wavelengths at room temperature. The solution concentrations were $10 \mathrm{mg} \mathrm{mL}^{-1}$. 
values of solutions with 0.1 and $10 \mathrm{mg} \mathrm{mL}^{-1}$ are 0.18 and $5.32 \%$, respectively. The emission intensity grows with an increase in concentration. To gain more information on the AIE process, the emission spectra of PSSMA aqueous solution and tetrahydrofuran (THF)/water mixtures were measured (Fig. S3, ESI $\dagger$ ). THF was employed because it was a non-solvent for PSSMA, and, thus, the polymer chains may be aggregated in the mixture with high THF fractions. When the THF fraction $\left(f_{\text {THF }}\right)$ is $0 \%$, only a weak signal is recorded. However, when $f_{\mathrm{THF}}$ has increased to $99 \%$, the emission intensity rapidly increases as well due to molecular aggregation leading to a strong blue emission at $\sim 380 \mathrm{~nm}$. These results indicate that aggregates are formed when the nonsolvent content is increased and the emission intensity is enhanced; therefore, PSSMA is an AIE-active polymer.

\subsection{Excitation dependent fluorescence emission}

Many reported polymer materials and carbon dots exhibit emission dependence on the excitation wavelengths. ${ }^{32-34}$ The fluorescence emission spectra of PSSMA aqueous solution (10 $\mathrm{mg} \mathrm{mL}^{-1}$ ) and solids samples were measured at different excitation wavelengths to assess the presence of such dependence. The excitation-wavelength dependence fluorescence emission is evident in Fig. 2; there, the emission peak wavelength of PSSMA aqueous solutions is red-shifted from $360 \mathrm{~nm}$ to $465 \mathrm{~nm}$, while the emission peak wavelength of the solid powder is red-shifted from $360 \mathrm{~nm}$ to $470 \mathrm{~nm}$. Interestingly, the solution $\left(10 \mathrm{mg} \mathrm{mL}^{-1}\right)$ emission intensity gradually decreases while the emission peaks red-shift as the excitation wavelength gradually increases from 300 to $400 \mathrm{~nm}$ (Fig. 2). However, by increasing the excitation wavelengths, the emission intensity of solid sample tends to increase first and then decrease. When excited at $380 \mathrm{~nm}$, the fluorescence intensity is at its maximum. Zn-PSSMA and Ca-PSSMA solid powder also exhibit excitationwavelength dependence fluorescence emission under different excitation wavelengths (Fig. S4, ESI $\dagger$ ). XRD spectra contain no sharp peaks (Fig. S5, ESI $\dagger$ ), which suggests the amorphous state nature. Therefore, the solid emission due to ion bond fixes the conformations, decreasing the thermal deactivations and promoting the light emissions from clustering-induced emission of carbonyl and sulfonic acid groups. The presence of the excitation-dependent fluorescence emission indicates that PSSMA solution and solids possess versatile optical properties.

\subsection{Room-temperature phosphorescence}

Carbonyl and sulfonic acid groups are beneficial for enhancing intersystem crossing and achieving phosphorescence emission. PSSMA, Zn-PSSMA, and Ca-PSSMA solid samples were taken under $312 \mathrm{~nm}$ UV lamp; a after the removal of the UV lamp, remarkable long green afterglow can be observed at second scale in both Zn-PSSMA and Ca-PSSM; these features are not present in the PSSMA sample (Fig. 3). These results indicate that Zn-PSSMA and Ca-PSSMA have RTP properties. To gain deeper insight into the photophysical properties of PSSMA, Zn-PSSMA, and Ca-PSSMA solids samples, the prompt and steady-state photoluminescence spectra were collected under ambient
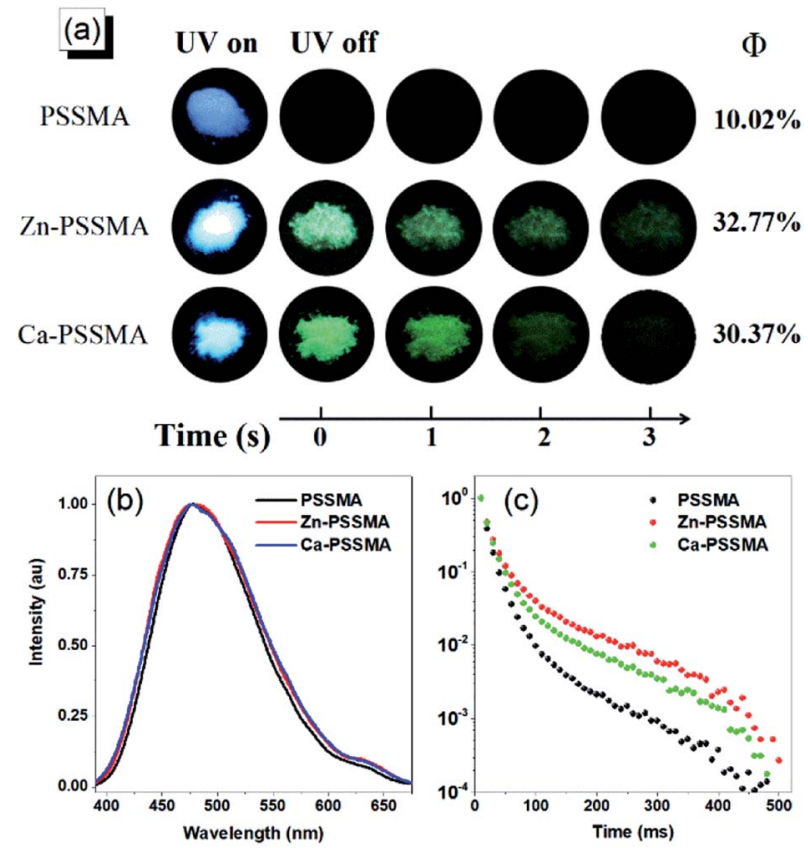

Fig. 3 (a) Pictures of PSSMA, Zn-PSSMA, and Ca-PSSMA under $312 \mathrm{~nm}$ UV source and different times after removal of the UV source. Phosphorescence spectra (b) and time-resolved measurements (c) of $480 \mathrm{~nm}$ emission from PSSMA, Zn-PSSMA, and Ca-PSSMA solid samples at room temperature. The excitation wavelength of spectrum is $320 \mathrm{~nm}$.

conditions. Besides, the time-resolved photoluminescence spectra were obtained delayed $10 \mathrm{~m} \mathrm{~s}$ after excitation, while emission-decay curves with different excitation wavelengths were measured; for these experiments, the delayed emission peaks were located at 480, 490, and $495 \mathrm{~nm}$, respectively. Under excitation wavelength of $320 \mathrm{~nm}$, the peaks of the prompt emission of the solution, solid powder, and solid delayed emission of PSSMA are located at 379, 381, and $480 \mathrm{~nm}$, respectively (Fig. 2 and $3 \mathrm{~b}$ ). The prompt and delayed emission peaks differ by $\sim 100 \mathrm{~nm}$, indicating that the delayed emission peaks are assigned to the phosphorescence emission phenomena.

The longest phosphorescence lifetime of PSSMA is $18.78 \mathrm{~m} \mathrm{~s}$, but the lifetime of Zn-PSSMA and Ca-PSSMA was extended to $40.64 \mathrm{~m} \mathrm{~s}$ and $28.54 \mathrm{~m} \mathrm{~s}$ (Fig. $3 \mathrm{~b}$ and $\mathrm{c}$ ). The sums of quantum efficiency of phosphorescence and fluorescence $(\Phi)$ of PSSMA, Zn-PSSMA, and Ca-PSSMA were 10.02\%, 32.77\%, and 30.37\%, respectively. High quantum efficiency and long lifetime of $\mathrm{Zn}$ PSSMA and Ca-PSSMA contribute to restricting the molecular motions (rotations and vibrations) of $\mathrm{Zn}^{2+}$ and $\mathrm{Ca}^{2+}$ ionic bonds via crosslink; these materials display persistent phosphorescent emission behavior at room temperature.

\subsection{Excitation-dependent phosphorescence emission}

The time-resolved (delayed) photoluminescence spectra $\left(t_{\mathrm{d}}=\right.$ $0.5 \mathrm{~m} \mathrm{~s}$ ) of solid powders (PSSMA, Zn-PSSMA, and Ca-PSSMA) with different excitation wavelengths were measured, and the heat maps were made at the same time. When excitation 

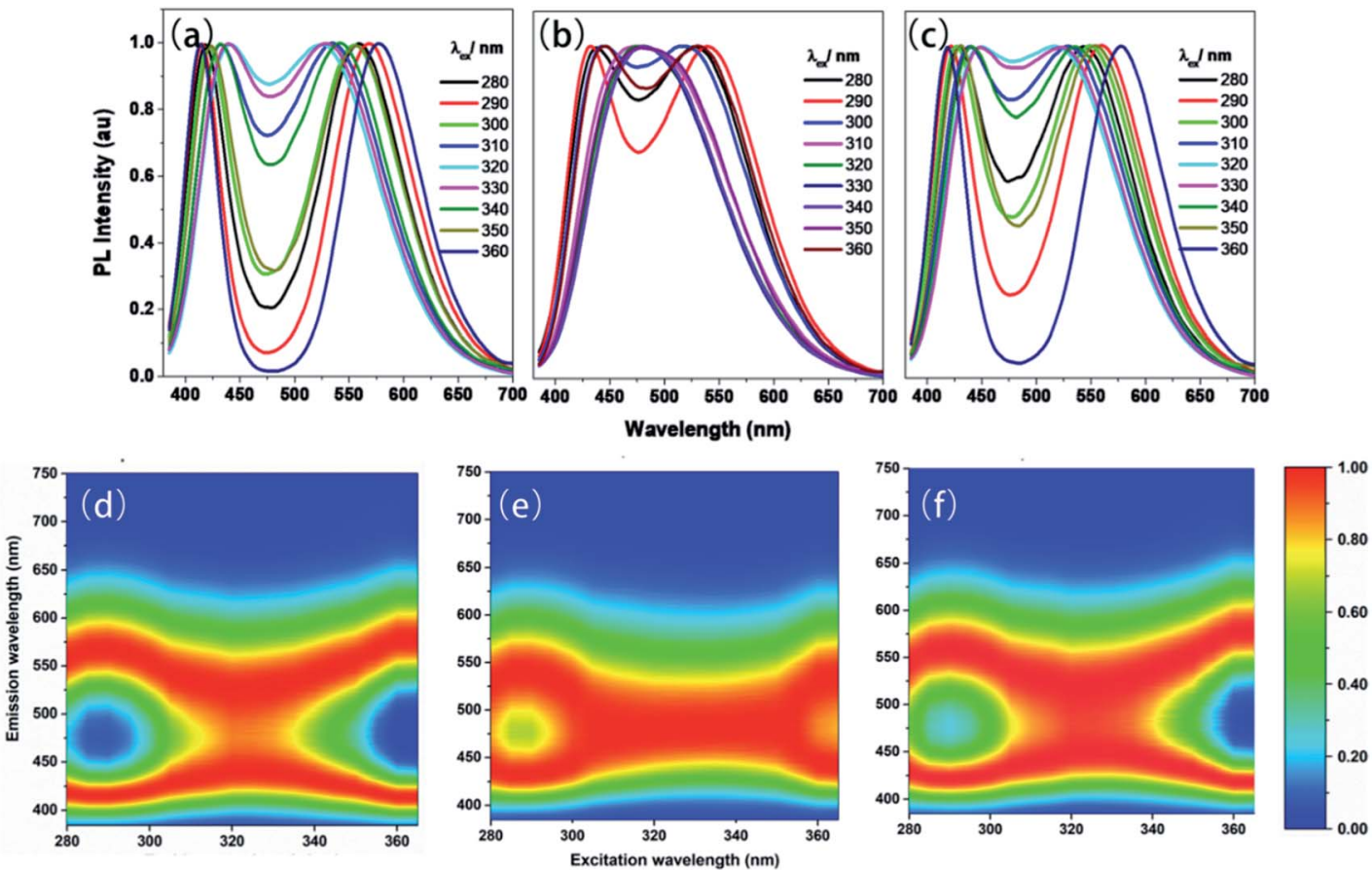

Fig. 4 Phosphorescence spectra $(a-c)$ with different excitations and excitation-phosphorescent emission heat maps (d-f) of PSSMA (a and d), Zn-PSSMA (b and e), and Ca-PSSMA (c and f) solid powders.

wavelength is $280 \mathrm{~nm}$, the photoluminescence spectra of these materials contain two peaks. As the excitation wavelength increases, the distance between two peaks shortens until coalescence. However, once the excitation wavelength has reached
320-330 $\mathrm{nm}$, the two peaks are separated again but with the opposite trend (Fig. 4). The excitation-wavelength dependence emission of the polymers is mainly due to the polydispersity of the high polymer amount and various condensed states that
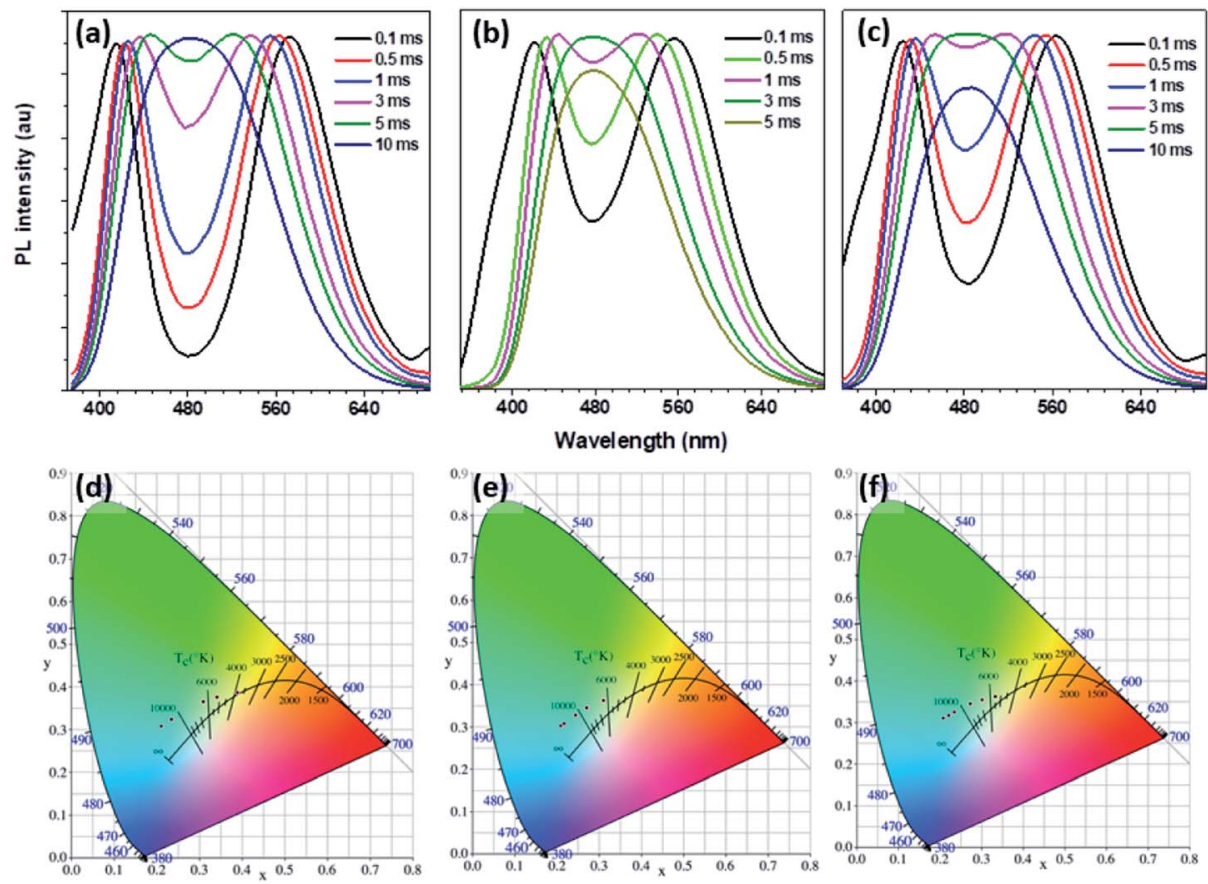

Fig. 5 Phosphorescent emission spectra with different delay times $(a-c)$ and counterpart CIE chromatic coordinates (d-f) of PSSMA (a and d), $\mathrm{Zn}$-PSSMA (b and e), and Ca-PSSMA (c and f) solid powders. The excitation wavelength of PSSMA, Zn-PSSMA, and Ca-PSSMA are 355, 290, and $350 \mathrm{~nm}$, respectively. 
form different excitation seeds. The strongest emission wavelength of these excited seeds is closely related to the excitation wavelength. Thus, the excitation wavelength dependence of RTP is confirmed.

\subsection{Delay-time dependent phosphorescence emission}

We found that the RTP emission of these materials has delaytime dependent characteristics. The time-resolved RTP spectra of PSSMA, Zn-PSSMA, and Ca-PSSMA solid powders with different delay times were recorded. All the phosphoresces spectra as well as the CIE chromatic coordinates were illustrated in Fig. 5. By increasing the delay time, the spectrum profile changes with the two emitting peaks becoming a single emitting peak positioned at $\sim 480 \mathrm{~nm}$; at same time, the CIE coordinates (Tables S1 and S2, ESI $\dagger$ ) have varied significantly, leading to a color variation as well. These results suggest that these RTP emissions possess delay time dependence properties. To explain the time dependence of the phosphorescence emission the free volume theory proposed by Fox and Flory can be used. According to this theory, the volume of a polymer consists of two parts: one is the volume occupied by the molecule, called the occupied volume, while the other is the unoccupied free volume and is dispersed throughout the material in the form of "holes". It is because of the existence of the free volume that it provides space for changing the conformation for the relaxation of the excited states. Delay-time dependent phosphorescence emission due to the conformation and energy level between $T_{1}$ and $S_{0}$ changes over time. It is worth noting that the RTP emission of PSSMA also has a delay time dependence under different excitation wavelengths (Fig. S6, ESI †).

\section{Conclusions}

In conclusion, halogen- and noble-metal-free polymer materials based on commercialized PSSMA possess aggregation-induced emission and RTP characteristics. The excitation intensity, excitation wavelength, and temperature dependence of photoluminescence, especially fluorescence, have been previously reported in literature. However, this work focused on the delaytime-dependent RTP emission for the first time in literature. This phenomenon is due to excited state relaxation dynamics with a slower transition rate from the excited triplet state to the ground state. In this process, the triplet excited state may be unstable, and the molecular conformation of the triplet excited state changes with time, causing energy level changes and the time dependence of RTP emission. Due to their unique optical properties, these polymers may be widely used in bioimaging, sensing, optoelectronic devices, and so on. The photophysical processes and potential applications of these polymers will be thoroughly studied and reported in due course.

\section{Conflicts of interest}

There are no conflicts to declare.

\section{Acknowledgements}

This work was financially supported by the National Natural Science Foundation of China $(51603050,51863006)$, the Natural Science Foundation of Guangxi (2016GXNSFBA380196 and 2016GXNSFBA380064).

\section{Notes and references}

1 Y. Xie, Y. Ge, Q. Peng, C. Li, Q. Li and Z. Li, Adv. Mater., 2017, 29, 1606829.

2 W. Zhao, Z. He, J. W. Lam, Q. Peng, H. Ma, Z. Shuai, G. Bai, J. Hao and B. Z. Tang, Chem, 2016, 1, 592-602.

3 Y. Zhou, W. Qin, C. Du, H. Gao, F. Zhu and G. Liang, Angew. Chem., Int. Ed., 2019, 58, 12102-12106.

4 S. Mukherjee and P. Thilagar, Chem. Commun., 2015, 51, 10988-11003.

5 N. Gan, H. Shi, Z. An and W. Huang, Adv. Funct. Mater., 2018, 28, 1802657.

6 G. Zhan, Z. Liu, Z. Bian and C. Huang, Front. Chem., 2019, 7, 305.

7 G. He, L. Du, Y. Gong, Y. Liu, C. Yu, C. Wei and W. Z. Yuan, ACS Omega, 2019, 4, 344-351.

8 W. Z. Yuan, X. Y. Shen, H. Zhao, J. W. Y. Lam, L. Tang, P. Lu, C. Wang, Y. Liu, Z. Wang, Q. Zheng, J. Z. Sun, Y. Ma and B. Z. Tang, J. Phys. Chem. C, 2010, 114, 6090-6099.

9 Y. Gong, Y. Tan, H. Li, Y. Zhang, W. Yuan, Y. Zhang, J. Sun and B. Z. Tang, Sci. China: Chem., 2013, 56, 1183-1186.

10 C. Li, X. Tang, L. Zhang, C. Li, Z. Liu, Z. Bo, Y. Q. Dong, Y. H. Tian, Y. Dong and B. Z. Tang, Adv. Opt. Mater., 2015, 3, 1184-1190.

11 C.-R. Wang, Y.-Y. Gong, W.-Z. Yuan and Y.-M. Zhang, Chin. Chem. Lett., 2016, 27, 1184-1192.

12 J. Wei, B. Liang, R. Duan, Z. Cheng, C. Li, T. Zhou, Y. Yi and Y. Wang, Angew. Chem., Int. Ed., 2016, 55, 15589-15593.

13 J. Yang, X. Zhen, B. Wang, X. Gao, Z. Ren, J. Wang, Y. Xie, J. Li, Q. Peng, K. Pu and Z. Li, Nat. Commun., 2018, 9, 840.

14 S. Hirata, K. Totani, J. Zhang, T. Yamashita, H. Kaji, S. R. Marder, T. Watanabe and C. Adachi, Adv. Funct. Mater., 2013, 23, 3386-3397.

15 R. Kabe, N. Notsuka, K. Yoshida and C. Adachi, Adv. Mater., 2016, 28, 655-660.

16 R. Kabe and C. Adachi, Nature, 2017, 550, 384.

17 S. Reineke, N. Seidler, S. R. Yost, F. Prins, W. A. Tisdale and M. A. Baldo, Appl. Phys. Lett., 2013, 103, 093302.

18 H. Mieno, R. Kabe, N. Notsuka, M. D. Allendorf and C. Adachi, Adv. Opt. Mater., 2016, 4, 1015-1021.

19 D. Li, X. Yang and D. Yan, ACS Appl. Mater. Interfaces, 2018, 10, 34377-34384.

20 X. Qiao, Y. Han, D. Tian, Z. Yang, J. Li and S. Zhao, Sens. Actuators, B, 2019, 286, 1-8.

21 E. Lucenti, A. Forni, C. Botta, L. Carlucci, C. Giannini, D. Marinotto, A. Previtali, S. Righetto and E. Cariati, J. Phys. Chem. Lett., 2017, 8, 1894-1898.

22 Z. An, C. Zheng, Y. Tao, R. Chen, H. Shi, T. Chen, Z. Wang, H. Li, R. Deng and X. Liu, Nat. Mater., 2015, 14, 685. 
23 D. Chaudhuri, D. Li, Y. Che, E. Shafran, J. M. Gerton, L. Zang and J. M. Lupton, Nano Lett., 2010, 11, 488-492.

24 H. Ma, W. Shi, J. Ren, W. Li, Q. Peng and Z. Shuai, J. Phys. Chem. Lett., 2016, 7, 2893-2898.

25 H. Ma, Q. Peng, Z. An, W. Huang and Z. Shuai, J. Am. Chem. Soc., 2019, 141, 1010-1015.

26 Z. Y. Zhang, Y. Chen and Y. Liu, Angew. Chem., 2019, 131, 6089-6093.

27 Z. Cheng, H. Shi, H. Ma, L. Bian, Q. Wu, L. Gu, S. Cai, X. Wang, W. w. Xiong and Z. An, Angew. Chem., Int. Ed., 2018, 57, 678-682.

28 S. Cai, H. Shi, D. Tian, H. Ma, Z. Cheng, Q. Wu, M. Gu, L. Huang, Z. An, Q. Peng and W. Huang, Adv. Funct. Mater., 2018, 28, 1705045.

29 Y. Gong, L. Zhao, Q. Peng, D. Fan, W. Z. Yuan, Y. Zhang and B. Z. Tang, Chem. Sci., 2015, 6, 4438-4444.

30 H. Shi, Z. An, P.-Z. Li, J. Yin, G. Xing, T. He, H. Chen, J. Wang, H. Sun and W. Huang, Cryst. Growth Des., 2016, 16, 808-813. 31 H. Liu, Y. Gao, J. Cao, T. Li, Y. Wen, Y. Ge, L. Zhang, G. Pan, T. Zhou and B. Yang, Mater. Chem. Front., 2018, 2, 18531858.

32 D. Lee, O. Bolton, B. C. Kim, J. H. Youk, S. Takayama and J. Kim, J. Am. Chem. Soc., 2013, 135, 6325-6329.
33 X. Ma, C. Xu, J. Wang and H. Tian, Angew. Chem., Int. Ed., 2018, 57, 10854-10858.

34 T. Ogoshi, H. Tsuchida, T. Kakuta, T. a. Yamagishi, A. Taema, T. Ono, M. Sugimoto and M. Mizuno, Adv. Funct. Mater., 2018, 28, 1707369.

35 L. Du, G. He, Y. Gong, W. Z. Yuan, S. Wang, C. Yu, Y. Liu and C. Wei, Compos. Commun., 2018, 8, 106-110.

36 X. Qin, S. Wang, L. Luo, G. He, H. Sun, Y. Gong, B. Jiang and C. Wei, RSC Adv., 2018, 8, 31231-31236.

37 L. Pan, S. Sun, A. Zhang, K. Jiang, L. Zhang, C. Dong, Q. Huang, A. Wu and H. Lin, Adv. Mater., 2015, 27, 77827787.

38 H. Wang, C. Sun, X. Chen, Y. Zhang, V. L. Colvin, Q. Rice, J. Seo, S. Feng, S. Wang and W. W. Yu, Nanoscale, 2017, 9, 1909-1915.

39 A. Sharma, T. Gadly, A. Gupta, A. Ballal, S. K. Ghosh and M. Kumbhakar, J. Phys. Chem. Lett., 2016, 7, 3695-3702.

40 L.-L. Du, B.-L. Jiang, X.-H. Chen, Y.-Z. Wang, L.-M. Zou, Y.-L. Liu, Y.-Y. Gong, C. Wei and W.-Z. Yuan, Chin. J. Polym. Sci., 2019, 37, 409-415.

41 L. Gu, H. Shi, L. Bian, M. Gu, K. Ling, X. Wang, H. Ma, S. Cai, W. Ning and L. Fu, Nat. Photonics, 2019, 13, 406.

42 R. Rusakowicz and A. C. Testa, J. Phys. Chem., 1968, 72, 26802681. 